\title{
Lapurdum
}

Euskal ikerketen aldizkaria | Revue d'études basques |

Revista de estudios vascos | Basque studies review

$4 \mid 1999$

Numéro IV

\section{Les basques et le Pays Basque d'après La Nouvelle Géographie Universelle d'Elisée Reclus}

\section{Pierre Laborde}

\section{(2) OpenEdition \\ Journals}

Édition électronique

URL : http://journals.openedition.org/lapurdum/1576

DOI : 10.4000/lapurdum.1576

ISSN : 1965-0655

\section{Éditeur}

IKER

Édition imprimée

Date de publication : 1 octobre 1999

Pagination : 295-302

ISBN : 2-84127-156-0

ISSN : $1273-3830$

Référence électronique

Pierre Laborde, «Les basques et le Pays Basque d'après La Nouvelle Géographie Universelle d'Elisée Reclus », Lapurdum [En ligne], 4 | 1999, mis en ligne le 01 avril 2010, consulté le 30 janvier 2020. URL http://journals.openedition.org/lapurdum/1576 ; DOI : 10.4000/lapurdum.1576 


\section{Pierre LABORDE}

\section{LES BASQUES ET LE PAYS BASQUE D'APRÈS LA NOUVELLE GÉOGRAPHIE UNIVERSELLE D'ELISÉE RECLUS}

Elisée Reclus (1830-1905) a rédigé, presque seul, les 19 gros volumes de la "Nouvelle Géographie Universelle" publiés en moins de vingt ans, entre 1875 et 1880 sur l'Europe et entre 1880 et 1894 pour le reste du monde. Cette ouvre immense, qui représente 17873 pages et 4290 cartes, a fait de lui un géographe renommé dans le monde entier. Elle succède au "Précis de Géographie Universelle ou Description de toutes les parties du monde » écrit par Conrad Malte-Brun au début du siècle, à partir de 1810 ; également très connu, il est cependant moins novateur du point de vue de l'analyse géographique.

Pour mener à bien son entreprise, Elisée Reclus a beaucoup séjourné à l'étranger, s'est souvent déplacé en France car il a également participé à la rédaction des guides touristiques Joanne, et il appuyait aussi ses observations sur des lectures', ne pouvant tout voir par lui-même. On peut légitimement penser qu'il connaissait particulièrement bien le sud-ouest de la France car s'il est né à Sainte-Foy-la-Grande en Gironde, trois de ses frères sont nés à Orthez, l'un était également géographe, l'autre médecin et chirurgien et le troisième marin ${ }^{2}$. Cette familiarité avec cette partie de la France explique peut-être une des raisons pour laquelle, à la différence des autres géographes qui ont écrit sur la France avant ou après lui, Elisée Reclus commence son étude régionale par « Les Pyrénées, Les Landes et le bassin de la Garonne » et non par Paris et le bassin Parisien. Dans son ouvrage, les Basques et le pays Basque y ont une place convenable. Il en est question à deux reprises : la première dans un paragraphe intitulé «Ancêtres des Français » du premier chapitre: "Vue d'ensemble. Le milieu et la race $^{3}$ ", où il consacre une trentaine de lignes à l'ancienneté du peuplement basque et la seconde dans le deuxième chapitre, où il complète, en six pages, la présentation d'un point de vue géographique.

« Basques, Béarnais et Gascons se distinguent facilement des autres Français par leurs traits d'origine : on connaît leur grâce naturelle, leur souplesse de corps et d'esprit, leur gaieté dans le travail, leur bravoure mêlée de forfanterie, leur facilité d'élocution, leur langage caressant, leur imagination vive, qui les emporte souvent au delà des limites du vrai. $»^{4}$

Ce portrait, avantageux mais pas totalement flatteur, où Basques, Béarnais et Gascons se trouvent associés comme au temps des Vascons, ne doit pas masquer le sérieux et l'aspect scientifique de son ouvrage. Cette distinction par rapport aux autres Français, Elisée Reclus l'attribue à une différence d'origine. La France n'était-elle pas peuplée de Celtes comme l'avait déjà remarqué Jules César alors que les peuples d'Aquitaine ${ }^{5}$ étaient Ibères selon Elisée Reclus. 
«Au nord des Pyrénées, ces représentants de la race des Ibères occupaient l'espace limité par la Garonne et l'océan Atlantique... » ${ }^{6}$

Mais, ils avaient occupé un territoire plus vaste si l'on en croît la toponymie :

«Les noms de lieux des Pyrénées françaises, dans toute la partie occidentale et même à l'extrémité orientale de la chaîne, sont ibériens et romains, non celtiques : ce qui fait supposer qu'avant la conquête des Gaules méridionales par les Romains les habitants du pays étaient les uns et les autres de langue euskarienne : il resterait à dresser la liste complète de tous les noms incontestablement basques de la région pyrénéenne... ${ }^{\top}$

Cette observation, bien connue et que les travaux de $J$. Corominas attestent, de la réduction de l'aire proto-bascophone, exprime aussi de manière indiscutable la continuité de l'usage de la langue basque dans un domaine beaucoup plus restreint et elle démontre, par conséquent, sa capacité de résistance.

«On sait que, par un remarquable phénomène historique, les populations ont gardé leur langue ibérique, d'ailleurs bien transformée non dans les régions les plus âpres, dans les bassins les plus fermés de la chaîne pyrénéenne, mais au contraire dans les vallons d'accès facile qui séparent les grandes Pyrénées des massifs de la côte cantabre ${ }^{8}{ }^{8}$

Cette thèse basco-ibérique, qui fait des Basques les héritiers des Ibères était normale à son époque quoiqu'elle fût déjà contestée ; aujourd'hui, cette parenté n'est plus guère retenue. Les progrès de la connaissance scientifique, à la suite de nombreux travaux de linguistique et de la découverte de sources inédites surtout à partir de 1918, ont eu raison de cette méprise entre les Basques et les Ibères ; Elisée Reclus constate toutefois que cette langue ibérique s'est modifiée. Elisée Reclus admire

« le noble idiome des Euskariens... ${ }^{9}$ qui est à proprement parler, le seul vraiment «européen » ou autochtone de l'Europe... ${ }^{10}$ »

cette seule langue non indo-européenne ancienne qui subsiste en Europe, et qui assure à ses locuteurs un haut lignage

« les Basques seraient les représentants de la race la plus ancienne du continent $» "$

Cependant, Elisée Reclus ne cache pas qu'un certain nombre de forces jouent en défaveur du maintien de l'euskara dans son cadre actuel. La plus importante est certainement la constitution de l'unité française :

«La communauté des épreuves et des malheurs, la prépondérance naturelle qu’a prise la capitale... enfin et surtout l'influence d'une langue littéraire rapprochant les idées différentes par une forme identique ont travaillé de concert à la constitution de l'unité française.... $\rangle^{12}$

Il n'emploie pas l'expression de la nation française bien qu'il définisse cette unité comme une communauté cimentée par une histoire commune, entraînée par la capitale et rassemblée autour d'une langue. Mais, cette unité ou cette nation n'est cependant pas totalement réalisée comme le prouve le cas de la Bretagne, des Flandres et du pays Basque « qui ont même partiellement gardé leur langue distincte ». D'autres motifs de recul de l'usage de la langue basque sont bien connus : l'instruction obligatoire joue un rôle incontestable; les villes exercent une forte influence qui est cependant moins fréquemment avancée. Mais Elisée Reclus ne se borne pas à reprendre des arguments que tout le monde connaît, il énonce aussi des idées neuves pour son temps :

« les diversités locales sont déjà dominées par la conscience de l'unité supérieure, et l'influence des villes est depuis longtemps prépondérante. Même les divergences dé parti qui divisent les citoyens contribuent, par le mouvement des idées et des passions, à niveler les anciennes barrières de séparation entre les provinces... $\rangle^{13}$ 
Il estime que la pluralité des opinions politiques et l'apparition de nouvelles doctrines en ce $\mathrm{XIX}^{\mathrm{e}}$ siècle entraînent des clivages entre les forces politiques. Républicains et monarchistes, conservateurs et libéraux, socialistes ou encore marxistes et anarchistes transcendent les particularismes provinciaux. Elisée Reclus est, en effet, affilié à la $1^{\text {re }}$ Internationale et, libertaire convaincu, il est même un des théoriciens de l'anarchisme ce qui lui a valu d'être ignoré des milieux universitaires français jusqu'à ces dernières années ${ }^{14}$. Dans la citation cidessus, il a aussi la conviction de la primauté de l'incorporation dans un ensemble plus vaste et du rôle des villes - et de Paris en particulier - comme vecteurs des idées nouvelles.

\section{Enfin, il a bien compris quelle pourrait être l'évolution future :}

"Quand tous les indigènes parleront les deux langues, ils finiront par négliger celui des deux idiomes qui leur sera le moins utile (d'autant plus facilement qu'il sera) dépourvu de l'influence exceptionnelle que donne la domination politique " ${ }^{\text {is }}$

Ce constat ne lui inspire aucun regret et ne le conduit pas à un commentaire partisan sur le rôle oppressif de l'État, preuve que son engagement politique personnel ne transparaît pas dans ses écrits de nature scientifique. Cependant, quand il écrit

«La France d'autrefois disparaît peu à peu sous la France nouvelle, comme un de ces tableaux changeants où les physiciens font disparaître un paysage d'hiver sous les couleurs du printemps, $\gg^{16}$

il est possible d'estimer qu'il y voit un processus apparemment positif en opposant le printemps à l'hiver, métaphore qui n'est évidemment pas fortuite.

On peut remarquer qu'Elisée Reclus ne condamne pas l'analphabétisme qui sévit en pays Basque. Est-ce son rejet de toute autorité qui explique ce silence ? Mais, à cette époque, on ne songeait guère à la revendication d'une alphabétisation de la population dans sa propre langue, ou bien cette demande n'était pas encore exprimée avec suffisamment de force.

« actuellement, il faut le dire, ce qui protège le Basque avec le plus d'efficacité contre les envahissements du français, c'est l'ignorance dans laquelle se trouvent les populations : elles n'éprouvent guère le besoin d'agrandir leur horizon intellectuel par I'usage d'une langue plus policée que la leur. D'après les statistiques les plus récentes, une moitié des hommes et les deux tiers des femmes du pays euskarien sont complètement illettrés : les Basques occupent donc pour l'instruction le dernier rang parmi les Français. ${ }^{17}$

Mais sa position est plus originale pour ce qui concerne les caractères de la région où le basque s'est maintenu. Alors que certains groupes humains et linguistiques minoritaires ont généralement trouvé refuge dans des montagnes, observation faite traditionnellement aussi pour les Basques, Elisée Reclus ne la reprend pas. Effectivement, comparé aux Pyrénées centrales et à la cordillère cantabrique, le pays Basque apparaît comme un milieu ouvert. Ce concept d'ouverture ne fait que renforcer le particularisme et la vigueur de cette vieille langue puisqu'elle a su se maintenir dans un contexte perméable aux influences extérieures.

D'ailleurs, les Basques ne sont pas renfermés sur eux-mêmes ni repliés sur leurs terres comme pourrait le faire croire leur illettrisme.

«C'est principalement vers Bayonne, Toulouse et Bordeaux que se dirige l'exode 
des Basques allant se mêler aux populations gasconnes; des milliers d'Euskariens y travaillent en qualité d'arrimeurs, de portefaix, d'artisans, d'employés de commerce, tandis que les basquaises entrent dans les familles comme domestiques. ${ }^{18}$

Mais, c'est surtout à destination de l'Amérique que les départs sont nombreux ; cette émigration est le fait de

« jeunes hommes abhorrant pour la plupart le service militaire et d'ailleurs faciles à entraîner par l'amour des aventures lointaines, qui est chez eux un instinct de race et qui fit de leurs ancêtres de si hardis pêcheurs de baleines... Ces jeunes gens, à leur tour, entraînent après eux des parents et des amis, et c'est ainsi que le Nouveau Monde, au Venezuela, au Chili, surtout dans la république Argentine, contient maintenant plus de Basques français, émigrés ou fils d'émigrés que n'en contient la France ellemêrne. $)^{19}$

L'appel est si fort que, faute de main d'œuvre, note Elisée Reclus,

«il n'est pas rare de voir des champs abandonnés par leur propriétaire, même avant la récolte. $\${ }^{20}$

Mais, au-delà des raisons psychologiques qui entrent dans la motivation de la plupart des départs, Elisée Reclus n'évoque pas, à ce stade de son propos, ni cause économique (l'insuffisance des ressources, la faiblesse de l'emploi non agricole, l'artisanat frappé par la révolution industrielle et la diminution de la population...) ni motif social (le fort excédent naturel, la quasi-obligation des cadets de famille à partir, le régime de succession parentale...) pour expliquer l'intensité de l'émigration. Plus loin, il remarque cependant que

"la population ne trouve pas dans la contrée de ressources suffisantes, quoique le nombre moyen des habitants par kilomètre carré ne soit pas pourtant plus élevé que dans le reste de la France. $\gg^{21}$

La prise en compte du milieu naturel est, pour Elisée Reclus comme pour tous les géographes, un élément essentiel pour connaître les phénomènes humains mais il faut se garder de tout déterminisme physique; aussi reconnait-il que la conformation des pays tient autant à l'homme qu'à la nature. Ainsi, de l'influence espagnole, estime-t-il, qui l'emporte sur l'influence française :

« l'opposition des versants donne la raison de ce fait. Les gens de la montagne et du plateau, c'est à dire les Espagnols, avaient en effet, plus de facilité pour descendre dans la plaine que les habitants de la plaine n'en avaient pour escalader les monts. En outre, le versant français doit exercer une plus grande force d'attraction à cause de la richesse de ses pâturages, de la fertilité de ses vallées, de l'animation de ses villes. $\$^{22}$

Il faut, en outre, tenir compte des genres de vie qui s'opposent également :

«Les Pyrénéens du Nord sont naturellement plus attachés au sol, et leurs voisins du sud plus facilement nomades; les premiers sont presque tous agriculteurs, tand is que les Espagnols ont parmi eux une plus forte proportion de bergers : ce sont eux aussi qui fournissent le personnel d'intrépides contrebandiers. ${ }^{23}$

Ces deux groupes d'arguments et, en particulier, la pression des Espagnols expliquent que la frontière a été

« presque partout tracée à l'avantage des montagnards espagnols... et se trouve en grand désaccord avec les lignes naturelles de faîte. Si les bornes politiques suivaient l'arête de partage des eaux, la vallée de la Bidassoa serait française; de même le val Carlos, au nord de Roncevaux, le val d'Aran et beaucoup d'autres régions moins étendues cesseraient d'appartenir à l'Espagne. ${ }^{24}$ 
Comme elle n'est pas ici fondée sur le droit de la guerre, donc des États, Elisée Reclus la juge plus stable. Elisée Reclus souligne néanmoins le caractère «parfait » de cette frontière politique que renforcent l'absence de voies ferrées qui percent la chaîne et la rareté des routes carrossables qui la traversent de part en part. Elisée Reclus en tire une conclusion :

"Les Pyrénées se dressent encore en obstacle infranchissable entre les peuples. $»^{25}$

Au contraire de ses prédécesseurs, Elisée Reclus ne cherche pas à dresser un inventaire méthodique des fleuves, des rivières et des montagnes, des villes et des bourgs, à répertorier les cadres administratifs, à dénombrer les populations et à énumérer les productions. Il s'applique plutôt à rechercher l'explication des paysages et des ressources qu'il décrit.

« Le département des Basses-Pyrénées est surtout un pays agricole, du reste mal cultivé. Les touyas, qui fournissent la litière des animaux, sont des fourrés d'ajoncs, de fougères et de bruyères diverses, au milieu desquels croissent çả et là des chênes « tauzins » ; ils couvrent, sur les collines avancées... un espace énorme dépassant trois cent mille hectares. ${ }^{26}$

Il n'est pas le seul à dénoncer cette sous-occupation de vastes surfaces mais il ne l'explique pas alors que, à son époque et jusqu'au milieu du $\mathrm{XX}^{e}$ siècle, la lande entre dans le système d'exploitation agricole ou pastorale.

Les villages et les petites villes de l'intérieur ne font l'objet que d'une description fragmentaire parce que leur importance est limitée et leurs caractères sont simples :

«Les communes populeuses de l'intérieur du pays Basque, dans les vallées du Saison, de la Bidouze, de la Joyeuse, de la Nive et de la Nivelle, se composent pour la plupart de hameaux pittoresques épars au milieu des arbres... Tardets, sur le haut Saison, est devenu un grand et prospère atelier de fabrication de sandales. MauléonLicharre, ancienne capitale de la Soule et chef-lieu d'arrondissement... Hasparren, entourée de landes, a ses nombreux ateliers de cordonnerie, ses fabriques de drap. Saint-Jean-Pied-de-Port a sa petite forteresse... ${ }^{27}$

On peut s'étonner du traitement opposé de Tardets et de Mauléon, car, dans cette seconde moitié du XIX ${ }^{e}$ siècle, c'est évidemment Mauléon, qui est le grand centre de fabrication de sandales. Confusion ou mauvaise relecture ? Mais, dans cette description, une première appréciation esthétique apparaît sur les « hameaux pittoresques », il y en aura une seconde sur Bayonne qui a « un air de gaieté » et une troisième sur la «beauté des plages ».

L'analyse des faits économiques, sociaux et politiques de la Côte basque est plus complète pour une simple raison qui traduit bien l'orientation du développement amorcée à partir du milieu du XIXe siècle :

«La vie du pays Basque et de la basse vallée de l'Adour s'est portée principalement sur le littoral. $»^{28}$

En quelques mots, bien choisis et d'une grande justesse d'observations, Bayonne est très complètement décrite. On n'ignore rien de sa structure et de son paysage urbain :

"Quoique forte place de guerre, entourée de murs et dominée au nord par une puissante citadelle, Bayonne semble une ville ouverte, grâce à ses promenades, à ses places et aux larges espaces de lumière que lui ménagent vers l'horizon la Nive et l'Adour ; les maisons de la ville, peintes, à l'espagnole, de couleurs diverses, contribuent aussi à 
lui donner un air de gaieté ; sa cathédrale, édifice gothique du douzième et du treizième siècle, est un des plus remarquables édifices du midi de la France. » ${ }^{29}$

de son économie et de son développement qui sont conditionnés par des contraintes naturelles

«Comme cité maritime, Bayonne est encore loin d'avoir toute l'activité que semblerait devoir lui assurer sa position vers l'angle du golfe de Gascogne, entre Bordeaux et Bilbao : les périls de la mer de Biscaye, les obstacles si difficiles à surmonter que la barre d'entrée de l'Adour oppose aux navires »

qu'Elisée Reclus précise dans un paragraphe relatif à la côte des Landes :

« la barre de l'Adour est à bon droit redoutée des marins... ; l'embouchure est si étroite, que les navires ont à peine l'espace nécessaire pour en franchir le seuil... la passe est loin d'être fixée ; il est même arrivé, mais d'une manière tout exceptionnelle, que le courant fluvial s'est épanché en entier par-dessous les jetées, tandis qu'un banc de sable occupait toute la largeur de l'embouchure. $)^{30}$

Les impératifs physiques, qui ont été partiellement corrigés ou atténués par des travaux incessants et jamais définitifs, ne sont pas seuls en cause. Jouent aussi les stratégies des principaux acteurs de la cité : l'armée qui impose ses servitudes, les commerçants et les banquiers qui se détournent du port.

« les réglementations gênantes imposées par les chefs militaires, les avantages qu'ont en revanche les négociants à expédier par le chemin de fer leurs marchandises sur l'Espagne ou sur Bordeaux, enfin la direction que la colonie de Juifs espagnols et portugais, moins aptes au trafic maritime qu'aux affaires de banque, a donnée au commerce, n'ont laissé à Bayonne qu'un rang pcu élevé parmi les marchés de la France. »"31

\section{Elisée Reclus poursuit}

«Quant aux petits havres du littoral basque, Guéthary, Saint-Jean-de-Luz, ils n'ont plus aujourd hui la moindre importance commerciale et leurs marins si hardis n'ont plus à s'occuper que de la pêche. Et pourtant les ancêtres de ces hommes fréquentaient les côtes de l'Amcrique du Nord avant Colomb! Dans le pays Basque, la tradition unanime attribue la découverte du Nouveau Monde à un certain Echaïde, et cette tradition n'a rien que de plausible, puisque, dès le milieu du quinzième siècle, les cartes indiquent au loin dans l'Atlantique occidentale des îles des Bacalaos ou des « Morues » et que ce nom basque s'est conservé pour l'île de Terre-Neuve jusqu'à une époque récente. La désignation de Cap-Breton, empruntée à l'ancien port de l'Adour, a été appliquée par les marins eskuaras à la grande île du littoral de la Nouvelle-Écosse'. Il est vrai que bien avant les Basques les Scandinaves avaient déjà̀ visité les côtes septentrionales du continent américain. »

Plus personne ne conteste cette antériorité des Scandinaves et, dès 1470, les parages de Terre-Neuve sont fréquentés par des pêcheurs basques, bretons ou britanniques. Mais ils ne se doutent pas qu'ils sont aux abords d'un nouveau continent. Ils n'ont pas «inventé l'Amérique »"

«Actueliement on travaille à la prolongation de la jetée de Socoa pour la rattacher au banc d'Arta; à l'entrée de la baie de Saint-Jean-de-Luz, et garantir ainsi les navires de la houle du large ; mais dût cette œuvre être menée à bonne fin, l'ancien port de Luz ou Lohitzun, vers lequel ne convergent point les routes commerciales, ne reprendrait point son ancien rang maritime. ${ }^{33}$

Au total, Elisée Reclus dresse un bilan plutôt moyen de l'activité maritime : le port de Bayonne est peu important concurrencé par le chemin de fer et insuffisamment utilisé, la pêche s'est replié sur le golfe de Gascogne. Alors, quel est l'avenir pour cette côte? Elisée Reclus pense au tourisme et à la pratique des bains 
de mer, qui a pris déjà de l'importance à Biarritz et qui pourrait le devenir pour Hendaye dont les premiers projets de transformation viennent d'être annoncés.

«L'importance de ces villes du littoral est maintenant dans la beauté de leurs plages lavées par le flot marin. C'est là ce qui a fait la rare prospérité de Biarritz, la ville de bains cosmopolite ; c'est là ce qui transformera peut-être le village d'Hendaye, si bien situé entre la Bidassoa et la mer, en face de Fontarabie et de son âpre montagne. " $^{34}$

La lecture des pages consacrées aux Basques et au pays Basque par Elisée Reclus donne une vision qui, aujourd'hui, n'est plus tout à fait inédite. Tout le monde partage l'idée que l'identité linguistique du pays Basque est tellement forte qu"elle constitue à elle seule l'argument autour duquel se définit tout le reste, à savoir toutes les valeurs, les modes de penser et les comportements sociaux. La cohérence de ce territoire ne vient donc ni de son cadre naturel ni de son économie. C'est pourquoi la transformation des mentalités et des pratiques culturelles sous des influences extérieures de plus en plus pressantes peut mettre en péril ce particularisme en l'absence de toute réaction.

L'information d'Elisée Reclus reste souvent riche et certaines idées sont, pour son époque, nouvelles et parfois prémonitoires; ses descriptions sont de qualité même si naturellement certaines pages ont vieilli mais il faut se garder de faire preuve d'anachronisme. Le succès de sa Géographie Universelle fut si grand que d'autres auteurs reprendront par la suite son argumentation.

Pierre LABORDE Université de BORDEAUX III 


\section{Notes}

1. S’agissant du pays Basque, Elisće Reclus a probablement lu « Le pays basque : sa population, sa langue, ses mœurs, sa littérature et sa musique » de Francisque-Michel qui a publié en 1857 cet ouvrage.

2. Onésime Reclus (1834-1916) a publié notamment La Terre à vol d'oiseau. Paul Reclus (1847-1914) fut un des premiers médecins à utiliser la cocaïne comme anesthésiant et Armand Reclus (1843-1927) est connu comme un des promoteurs du canal de Panama. Voir G. Fauconnier. Le lieutenant de vaisseau Armand Reclus et le canal de Panama. Atlantica, septembre 1996, p. 3-7.

3. Le mot de race est pris au sens ancien de groupe humain qui a des caractères semblables.

4. E. Reclus. Nouvelie Géographie Universelle. La Terre et les Hommes. Livre I L'Europe méridionale. Volume II La France. Paris, Hachette, 1885. Nouvelle éd. revue et corrigée. p. 45.

5. Il s'agit de la première Aquitaine antique dont les limites ne correspondent pas à l'Aquitaine actuelle.

6. E. Recius, op. cit. p. 45. Cet espace correspond grossièrement à l'antique Novempopulanie.

7. Ibid. p. 92.

8. Ibid. p. 92 .

9. Ibid. p. 93.

10. Ibid. p. 92.

11. Ibid. p. 92.

12. Ibid. p. 55.

13. p. 55 .

14. B. Giblin. 'Elisée reclus : géographie, anarchisme'. Hérodote, I976, p. 30-49. Y. Lacoste. 'Elisée Reclus : géographicité et géopolitique' in Paysages politiques. Paris, Libraire générale française, 1991 p. 191-233. "Elisée Reclus : un géographe libertaire". Hérodote, 1981, p...?

15. E. Reclus, op. cit. p. 94

16. Ibid. p. 55.

17. Ibid. p. 94

18. Ibid.p. 94

19. Ibid.p. 94.

20. Ibid. p. 94.

21. Ibid p. 153.

22. Ibid. p. 94

23. Ibid. p. 94.

24. Ibid. p. 95.

25. lbid. p. 96 .

26. Ibid p. 153.

27. Ibid. p. 153.

28. Ibid. p. 155.

29. Ibid. p. 155.

30. Jbid. p. 118 .

3!. Ibid. p. 155.

32. Th. Gomez. L'invention de l'Amérique. Mythes et réalités de la conquête. Paris, Aubier, 1992.

33. E. Reclus, op. cit. p. 156.

34. Ibid. p. 157. 